\title{
Efficacy of Ozone and Selenium Therapy for Alcoholic Liver Injury: An Experimental Model
}

\author{
YASEMEN ADALI ${ }^{1}$, HÜSEYIN AVNI EROǦLU ${ }^{2}$, MUSTAFA MAKAV ${ }^{3}$ and GÜLNAME FINDIK GUVENDI ${ }^{4}$ \\ ${ }^{1}$ Department of Pathology, Çanakkale Onsekiz, Mart University Faculty of Medicine, Çanakkale, Turkey; \\ ${ }^{2}$ Department of Physiology, Çanakkale Onsekiz Mart University Faculty of Medicine, Çanakkale, Turkey; \\ ${ }^{3}$ Faculty of Veterinary Medicine, Kafkas University, Kars, Turkey; \\ ${ }^{4}$ Department of Pathology, Rize Recep Tayyip Erdoğan University Faculty of Medicine, Rize, Turkey
}

\begin{abstract}
Background/Aim: Alcoholic liver disease is an important health problem which is reversible during early stages of liver damage, but becomes permanent with time. Nowadays, many studies focus on various agents that prevent, reduce or slow the progression of the toxic effects of alcohol. In our study, we investigated the efficiency of ozone and selenium against oxidative damage in a model of alcohol-induced liver damage. Materials and Methods: Forty-eight female Wistar Albino rats between 4 and 6 months of age and weighing 190-250 $\mathrm{g}$ were included in the study and were used as models of alcohol liver damage. Aspartate aminotransferase (AST), alanine aminotransferase $(A L T)$, serum and tissue total oxidant levels, serum and tissue total antioxidant levels, and the histopathological evaluation of the liver were performed in 8 groups. Results: In the statistical analysis, it was observed that ozone and/or selenium therapies decreased the AST levels. Total oxidant and antioxidant serum levels were found to vary in serum and tissue. Ozone and/or selenium therapies decreased liver damage, according to histopathological findings. Conclusion: Through ozone and/or selenium therapies, less damage was observed histopathologically compared to the alcohol group. It is thought that the results of our study can be used in individual treatments following confirmation of liver damage in alcoholic patients.
\end{abstract}

Alcoholic liver disease is a spectrum of pathological

This article is freely accessible online.

Correspondence to: Yasemen Adali, Çanakkale Onsekiz Mart University Faculty of Medicine, Department of Pathology, Çanakkale, Turkey. Tel: +90 5063437199, e-mail: yasemenadali@ hotmail.com

Key Words: Alcoholic liver disease, ozone, selenium, experimental model. conditions commonly encountered, especially in western societies, and can result in liver cirrhosis or even hepatocellular carcinoma (1). Both excessive and long-term alcohol consumption may cause alcoholic liver disease (2). Even though alcohol is considered as hepatotoxic and more than $90 \%$ of people who consume alcohol excessively show signs of accumulated fat within their bodies, only $30 \%$ of these cases develop serious forms of alcoholic liver disease (3). Complex interactions of alcohol with gender, heredity and immunity have been implicated in the pathogenesis of the disease; however, their role still remains unclear (3). It is known that the metabolism of alcohol in the liver initiates a pathological process that includes the production of protein aldehyde compounds and endotoxins, oxidative stress, immunological activities and proinflammatory cytokine release. When all these parameters are included, steatosis (fatty liver), steatohepatitis and variable stages of fibrosis can be observed by histopathology (4). For the development of hepatic steatosis, which is the first stage of alcoholic liver disease, daily consumption of more than 60 grams of alcohol is adequate $(5,6)$. In alcoholic liver disease, histopathological features in the liver are still reversible, especially during early stages, however, as the disease progresses, these become irreversible and end up with liver fibrosis (7).

Ozone and selenium are agents that are clinically used in medicine and have antioxidant properties $(8,9)$. Ozone used as a mixture of $\mathrm{O}_{2} / \mathrm{O}_{3}$ in medical applications and is known to be involved in the management of antioxidant enzymes, nitrite oxide pathways and a large number of different cellular activities (10). In experimental studies, the positive effects of ozone in activating superoxide dismutase and reducing glutathione levels in acetaminophen-induced liver injury and liver ischemia reperfusion injury are reported (11, 12). Medical ozone gas, used in clinical practice, is produced in ozone generators and is used for the treatment of several diseases, such as in vascular disease affecting circulation, wound healing, viral diseases, such as hepatitis and AIDS and autoimmune diseases (13). Selenium is found in 
glutathione peroxidase, which is one of the most important antioxidant enzymes, and of selenoproteins. Selenoproteins are also important for a large number of immune system tasks, such as leukocyte and NK cell function, T lymphocyte proliferation and immunoglobulin production $(14,15)$.

In our study, we applied ozone and selenium therapy on experimentally induced alcoholic liver disease in rats, and evaluated their effect within the framework of oxidant, antioxidant and histopathological parameters.

\section{Materials and Methods}

This study was approved by the Kafkas University Experimental Animal Research Ethics Committee (Approval number: KAUHADYEK 2016/052). Animal procedures were performed according to the "Guide for the Care and Use of Laboratory Animals" principles (10). All steps of the study were conducted at the experimental research center of the university. Study reporting was done in accordance with the CONSORT principles (16).

Animals. Forty-eight female Wistar Albino rats between 4 and 6 months of age and weighing 190-250 g were included in the study, supplied from the Unit of Breeding Laboratory Animals at Atatürk University. The animals were fed ad-libitum and were kept at a temperature of $25^{\circ} \mathrm{C}$ in a place with a 12-hour light-dark cycle and ventilation. Animals were divided into 8 groups randomly, each consisting of 6 rats, as follows:

i) Group I: Control group which was fed ad-libitum without any applications.

ii) Group II: Rats in which alcoholic liver damage was induced by giving them $7 \mathrm{~g} / \mathrm{kg} /$ day ethyl alcohol $+50 \%$ water for 14 days.

iii) Group III: Rats in which intraperitoneal ozone was applied (95\% $\mathrm{O}_{2}+5 \% \mathrm{O}_{3}, 0.5 \mathrm{mg} / \mathrm{kg}$ ) for 7 days without inducing alcoholic liver damage.

iv) Group IV: Rats in which intraperitoneal selenium of $0.2 \mathrm{mg} / \mathrm{kg}$ was applied for 7 days without inducing alcoholic liver damage.

v) Group V: Rats in which both intraperitoneal selenium of $0.2 \mathrm{mg} / \mathrm{kg}$ and intraperitoneal ozone therapy $(95 \% \mathrm{O} 2+5 \% \mathrm{O} 3,0.5 \mathrm{mg} / \mathrm{kg})$ were applied for 7 days without inducing alcoholic liver damage.

vi) Group VI: Rats in which alcoholic liver disease was firstly induced by giving ethyl alcohol $7 \mathrm{~g} / \mathrm{kg} / \mathrm{day}+50 \%$ water for 14 days and then intraperitoneal ozone $\left(95 \% \mathrm{O}_{2}+5 \% \mathrm{O}_{3}, 0.5 \mathrm{mg} / \mathrm{kg}\right)$ was applied for 7 days.

vii) Group VII: Rats in which alcoholic liver disease was firstly induced by giving ethyl alcohol $7 \mathrm{~g} / \mathrm{kg} / \mathrm{day}+50 \%$ water for 14 days and then intraperitoneal selenium of $0.2 \mathrm{mg} / \mathrm{kg}$ was applied for 7 days, and

viii) Group VIII: Rats in which alcoholic liver disease was firstly induced by giving ethyl alcohol $7 \mathrm{~g} / \mathrm{kg} / \mathrm{day}+50 \%$ water for 14 days and both intraperitoneal selenium of $0.2 \mathrm{mg} / \mathrm{kg}$ and intraperitoneal ozone therapy $\left(95 \% \mathrm{O}_{2}+5 \% \mathrm{O}_{3}, 0.5 \mathrm{mg} / \mathrm{kg}\right.$ ) was applied for 7 days.

At the end of the study, the animals were not fed for one night and were anesthetised with ether, followed by cervical spine dislocation, in accordance with standards for ethical use and care of animals (17). Blood and liver samples of the animals were collected to be tested physiologically and biochemically and histopathologically, respectively.

Sample preparation. Following the collection of blood samples, some parts of the tissues were kept in $10 \%$ neutral buffered formalin for histopathological analysis. Following a wash with lactated ringer solution, the rest of the tissue samples were put into tagged polyethylene bags and were kept at $-20^{\circ} \mathrm{C}$ for future analysis. In accordance with the procedure of the kits (Total Oxidant Status Assay Kit, Total Antioxidant Status Assay Kit; REL Assay Diagnostics, Gaziantep-Turkey) used in the analyses, small parts of the liver tissue samples were weighed and homogenised through a homogeniser by adding phosphate buffer solution nine times heavier than the samples. Homogenates separated from the homogenized tissue samples by centrifugation were then taken into polyethylene tubes. Blood, tissue oxidant measurements were conducted in accordance with the manufacturers' instructions of assay kits (Total Oxidant Status Assay Kit, REL Assay Diagnostics, GaziantepTurkey), as well as the antioxidant measurements (Total Antioxidant Status Assay Kit; REL Assay Diagnostics, Gaziantep-Turkey).

Following tissue embedding, paraffin blocks were prepared and $4 \mu \mathrm{m}$ thick sections were stained with hematoxylin and eosin and were examined through a light microscope (Clinical Microscope Model BX46, Olympus, Japan) for fatty changes, inflammation, fibrosis, hepatocyte degeneration and congestion.

Statistical analyses. The SPSS 18 package program was used to evaluate the bio-statistical data obtained from the study. $t$-Test was used for independent variables in the 95\% Confidence Interval (CI), and $p<0.05$ was considered statistically significant.

\section{Results}

In our study, liver enzyme measurements, serum and tissue total oxidant status (TOS) and total antioxidant status (TAS) values and their distribution in the different groups are given in Table I. When these values were examined, we observed that aspartate aminotransferase (AST) and alanine transferase (ALT) increased in the alcohol group compared to the control group. In the ozone group, we found higher averages compared to both the control and the alcohol groups. The highest AST and ALT values were observed only in the ozone group, while the lowest values were observed in the alcohol + ozone group. It was noted that the AST values measured in the groups were lower compared to the alcohol and the control groups. Concerning the ALT values, we observed that the alcohol + selenium and the alcohol + ozone + selenium groups reached higher values compared to both the control and the alcohol groups. In addition, the AST values were found to be very close to each other in the alcohol + ozone and the alcohol + selenium groups, while the alcohol + ozone + selenium group had higher values. The statistical analysis revealed a level of significance between the control group and the alcohol + ozone group $(p=0.041)$. We observed that the application of ozone in the group of alcohol damage reduced the value of AST below the value of AST in the control group. In the alcohol group analysis, a statistical significance was observed between the alcohol + selenium $(p=0.007)$ and the alcohol + ozone + selenium $(p=0.007)$ groups in terms of the effects of different applications on decreasing AST values. When the substance applications in the alcohol groups were considered 
Table I. Mean AST, ALT, serum TOS, serum TAS, tissue TOS and tissue TAS values in the groups.

\begin{tabular}{lllrlll}
\hline Group No: & \multicolumn{1}{c}{ AST } & \multicolumn{1}{c}{ ALT } & Serum TOS & Serum TAS & TissueTOS & Tissue TAS \\
\hline 1 & $42.93 \pm 7.22$ & $137.52 \pm 13.94$ & $11.07 \pm 5.44$ & $1.63 \pm 0.29$ & $40.15 \pm 3.95$ & $15.99 \pm 3.78$ \\
2 & $48.97 \pm 3.37$ & $145.70 \pm 17.27$ & $7.58 \pm 1.38$ & $1.49 \pm 0.70$ & $37.91 \pm 3.06$ & $20.88 \pm 0.58$ \\
3 & $76.40 \pm 42.65$ & $438.29 \pm 367.28$ & $12.60 \pm 4.33$ & $1.40 \pm 0.21$ & $40.02 \pm 6.51$ & $16.62 \pm 1.25$ \\
4 & $51.57 \pm 5.07$ & $224.23 \pm 3.60$ & $7.00 \pm 2.68$ & $1.44 \pm 0.25$ & $38.38 \pm 1.91$ & $18.99 \pm 1.12$ \\
5 & $45.52 \pm 5.74$ & $209.52 \pm 73.23$ & $7.82 \pm 3.87$ & $1.44 \pm 0.12$ & $37.76 \pm 4.14$ & $91.44 \pm 178.60$ \\
6 & $34.95 \pm 4.20$ & $124.37 \pm 19.33$ & $10.70 \pm 1.71$ & $1.56 \pm 0.36$ & $41.17 \pm 1.91$ & $16.83 \pm 1.33$ \\
7 & $34.98 \pm 9.45$ & $185.40 \pm 30.75$ & $16.37 \pm 1.50$ & $1.48 \pm 0.12$ & $40.71 \pm 5.51$ & $16.80 \pm 1.81$ \\
8 & $41.67 \pm 5.28$ & $196.48 \pm 51.77$ & $10.09 \pm 1.55$ & $1.43 \pm 0.10$ & $40.89 \pm 4.83$ & $16.76 \pm 1.36$ \\
\hline
\end{tabular}

AST: Aspartate aminotransferase; ALT: alanine aminotransferase; TOS: total oxidant status; TAS: total antioxidant status.

among themselves, it was found that a statistical significance was found between the alcohol + ozone and the alcohol + selenium group $(p=0.002)$ and the alcohol + ozone + selenium group $(p=0.035)$ and that the alcohol + ozone group had lower AST values. In the statistical analyses of ALT, considering the values between the alcohol + selenium group $(p=0.006)$ and the alcohol + ozone + selenium group $(p=0.023)$, the control group had lower values. There were no statistically significant differences compared to the alcohol group. When the substance applications in the groups were considered among themselves, there was a statistical significance between the alcohol + ozone group and the alcohol + ozone + selenium group, favouring of the alcohol + ozone group $(p=0.01)$.

The serum TOS values of the control group were higher compared to the alcohol group. It was also observed that for the alcohol + substance the application values were higher compared to the alcohol group and that the highest value was in the alcohol + selenium group. While there was no difference between the control group and the other groups, there was a statistical significance between the alcohol group and the alcohol + selenium group $(p=0.000)$ and the alcohol + selenium groups $(p=0.004)$ in terms of lower values. When the alcohol and the alcohol + substance groups were compared, it was found that there was a significant difference between the alcohol + ozone and the alcohol + selenium groups in terms of lower values $(p=0.005)$ and that there was a statistical significance between the alcohol + selenium and the alcohol + ozone + selenium groups in favour of the alcohol + selenium group $(p=0.002)$.

The serum TAS values of the control group were higher compared to those of the other groups. It was observed that the alcohol + ozone application increased the value of serum TAS compared to the alcohol application and that the other substance applications showed lower values than the alcohol group. There was no significant statistical significance.

When the tissue TOS measurements were evaluated, the values of the alcohol group were found to be lower compared to the control group. When compared to the control group and the alcohol group, a high increase was observed in the values of all the alcohol + substance groups, but this was not statistically significant. When the alcohol + substance applications groups were compared between themselves, the highest value was observed in the alcohol + ozone group, but these differences were not significant either.

The ozone + selenium application in tissue TAS measurements was very high compared to the other groups. It was found that the alcohol application significantly increased the TAS average ( $p=0.011)$ compared to the control group and that there was a statistically insignificant increase in the alcohol + substance group compared to the control group. In addition, the alcohol group had statistically higher values than the alcohol + selenium group $(p=0.000)$ and the alcohol + ozone + selenium group $(p=0.000)$.

Histopathological examinations revealed that there were no symptoms apart from mild congestion in 4 rats and moderate congestion in 2 rats in the control group (Figure 1A). In the alcohol group, 1 rat had severe (Figure 1B), 3 rats had moderate (Figure 1C) and 2 rats had mild hepatocyte injuries, while the lesions were focal in 1 rat and widespread in 2 rats. In 2 rats, a mild hepatocyte injury was observed. A severe periportal inflammation was observed in 1 rat with a moderate focal hepatocyte injury and in 1 rat with a moderate injury (Figure 1D). In 6 rats of this group, varying levels of microvascular steatosis were observed (Figure 1E). The histopathological findings observed in the ozone group were similar to those of the control group (Figure 1F). In the selenium group, there was a mild periportal inflammation in 2 rats (Figure $1 \mathrm{G}$ ), while congestion was observed in all the other rats. In addition, a mild hepatocyte injury was observed in 1 rat. The findings observed in the ozone + selenium group were similar to those of the selenium only group and differed slightly as the mild hepatocyte injury was focal (Figure $1 \mathrm{H}$ ). In the alcohol + ozone group, 1 rat had a focal moderate hepatocyte injury (Figure 2A), whereas 1 rat had a widespread mild hepatocyte injury and 4 rats had a mild focal hepatocyte injury. In 2 rats, a mild periportal 

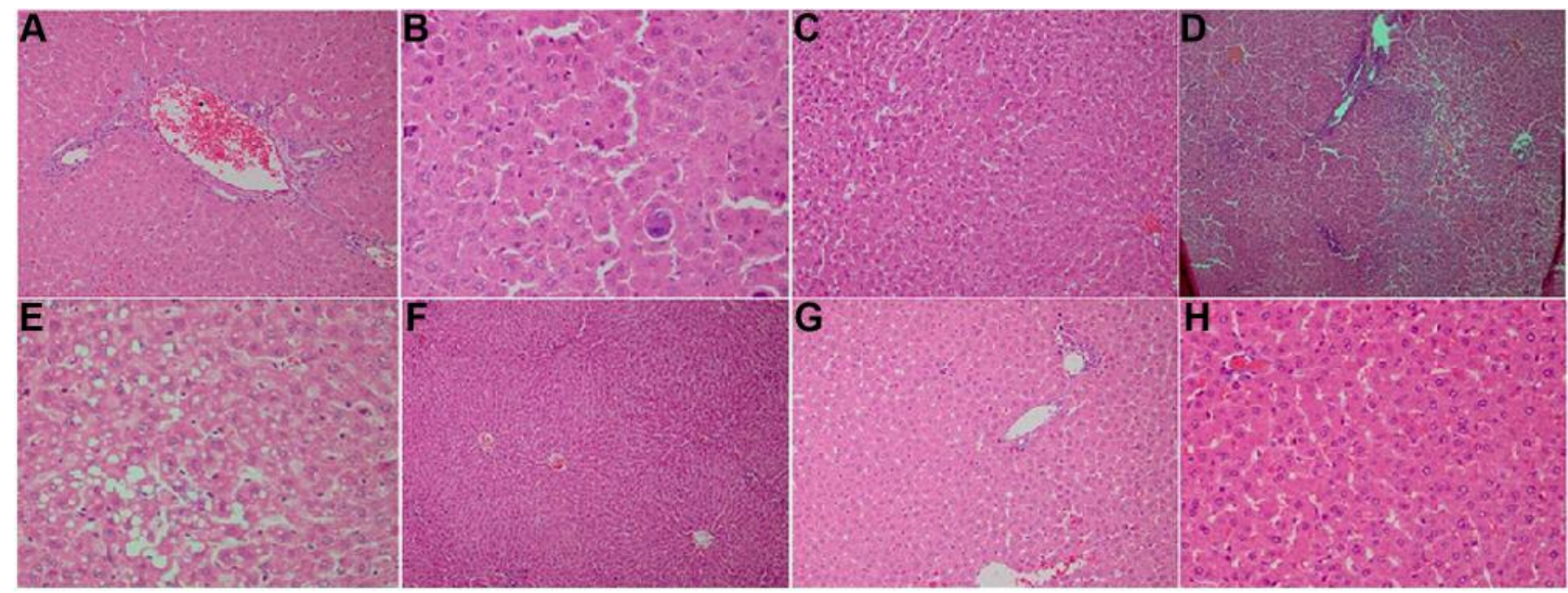

Figure 1. (A) Liver tissue with vascular congestion in the control group (H\&E, 200x). (B) Severe hepatocyte injury in the alcohol group (H\&E, $400 \times) .(C)$ Widespread moderate hepatocyte injury in the alcohol group $(H \& E, 200 \times)$. (D) Severe periportal inflammation in the alcohol group $(H \& E, 100 \times)$. (E) Microvesicular steatosis in the alcohol group $(H \& E, 400 \times)$. (F) Vascular congestion in the ozone group $(H \& E, 100 \times)$. (G) Mild periportal inflammation in the selenium group $(H \& E, 200 \times)$. (H) Focal hepatocyte injury in the ozone + selenium group $(H \& E, 100 \times)$.
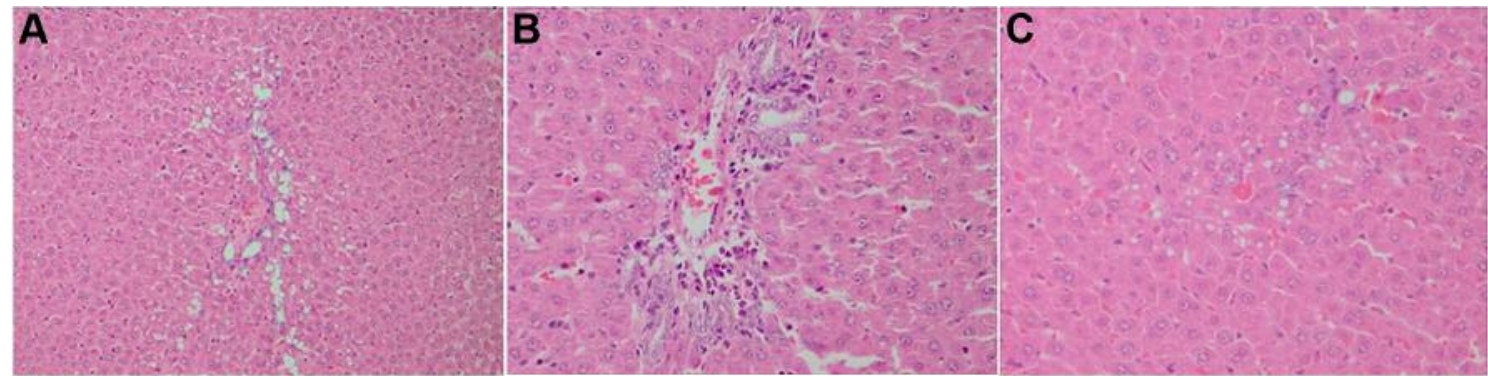

Figure 2. (A) Focal moderate hepatocyte injury and microvesicular steatosis in the alcohol+ozone group (H\&E, 200x). (B) Moderate periportal inflammation in the alcohol+selenium group $(H \& E, 400 \times)$. (C) Mild focal hepatocyte injury and microvesicular steatosis in the alcohol+ozone + selenium group $(H \& E, 400 \times)$.

inflammation was observed, whereas arying levels of microvascular steatosis were observed in four rats (Figure 2A), while microvascular steatosis was not observed in two rats. In the alcohol + selenium group, all the rats had varying levels of congestion and 5 rats exhibited a mild periportal inflammation (Figure 2B). Varying degrees of microvascular steatosis in all the rats and a mild hepatocyte injury in 3 rats ( 2 focal, 1 widespread) were observed. In the alcohol + ozone + selenium group we noticed a mild hepatocyte injury in 4 rats, a mild focal hepatocyte injury and varying degrees of microvascular steatosis in 3 rats (Figure 2C).

\section{Discussion}

Alcoholic liver disease can result in cirrhosis and hepatocellular carcinoma, lead to serious socioeconomic problems and reduce the quality of life (18). Early signs observed in liver might be steatosis or steatohepatitis or hepatitis (4). In our study, we evaluated the effects of the ozone and selenium applications on alcoholic liver damage using histopathological analyses and assessment of serum AST and ALT levels. It has been reported in various studies that AST and ALT levels increase independently of the cause of the damage (19-21). It has also been reported that selenium application with $\mathrm{N}$-acetylcysteine has a protective effect on lead and mercury toxicity $(19,20)$, lowers ALT activity in thioacetamide-induced cirrhosis and AST and ALT in arsenic toxicity (21). In the case of alcohol, it is reported that ebselen, an organoselenium compound, reduces elevated ALT in rats treated with alcohol (22). There are no studies on the use of ozone in alcohol-induced liver injury in the literature, but there are studies suggesting that the use of ozone in 
acetaminophen-induced liver injury decreases AST and ALT $(11,13)$. In addition, it is reported that ozone decreases AST and ALT in ischemia reperfusion injury (23) and that, in the experimental damage model created by radiation, ozone suppresses the rise of AST and ALT levels (24). In our study, we found that the ozone, selenium and ozone + selenium applications were effective in alcoholic liver injury in terms of AST, but the most effective application was the alcohol + ozone application. When we evaluated our ALT data, it was noted that in all the applications ALT had high values compared to the control group, and despite a numerical difference between the alcohol group and the substance applications, this was not reflected in the statistical analyses. When the alcohol and alcohol + substance groups were evaluated, the ozone application was the most effective of all. In this context, if we consider the AST and ALT values, we suggest that the most efficient substance is the use of ozone.

Oxidant and antioxidant mechanisms play an important role in liver damage induced by other toxic agents. Fatima SN et $a l$. have reported that selenium in thioacetamide-induced liver cirrhosis increases antioxidant enzyme levels (25). In another study, selenium in combination with $\mathrm{N}$-acetylcysteine was reported to reduce oxidative stress in mercury-induced liver damage (20), while selenium can also reduce oxidative stress when liver toxicity is induced by isotretinoin, an agent used for acne treatment (26). Similarly, selenium could reduce oxidative stress in carbontetrachloride-induced liver fibrosis when it was supplementing probiotic treatment (27). A different study reported that selenium can decrease cadmiuminduced hepatic and renal oxidative stress (28), however, Ghaffarian-Bahraman et al. pointed out that selenium only application was not adequately hepatoprotective in cadmium toxicity and that the application of both magnesium and selenium strengthened the protective effect of the latter (29).

Because ozone itself is oxidant, it is known that its lowdose application activates antioxidant mechanisms (10). In a study conducted by Gültekin et al., it has bben reported that ozone therapy provides organ protection by boosting the endogenous antioxidant defense mechanisms in radiationrelated organ damage (24), while, in another study Gültekin et al. suggested that ozone therapy can reduce the ischemiareperfusion injury in the liver of partially hepatectomized rats and that it could be used as hepatoprotective, due to its stimulating effect on hepatocyte regeneration (30). Ozone therapy has been found to have a positive effect on rat kidneys and liver by neutralizing age-related chronic oxidative stress (31), while it could decrease high MDA values and other antioxidant enzymes related to to acetaminophen damage (11). In our study, we observed that the use of alcohol decreased serum TOS values compared to the control group, while the drug application increased these values compared to the use of alcohol. We found that the alcohol + selenium application showed a statistical significance in the substance application and showed the highest TOS value. In addition, we found that the selenium and selenium +ozone applications to healthy liver showed a decrease in TOS values, similar to the alcohol application but with no statistically significant difference. Despite the fact that no statistical significance was observed when we compared TOS tissue values in our different models, the values of the alcohol group were lower compared to the control and the alcohol + substance groups. For this reason, we became sceptical about the dose of alcohol in our study that decreased oxidation and believe that there should be additional studies examining this issue in the future.

The results of our analyses on antioxidant values showed that the average of the serum TAS values in the control group was the highest among the groups. In the alcohol + ozone group, the serum TAS value was higher compared to the alcohol group; however, it was not the case in the other substance application groups. The analyses showed that this difference was not statistically significant, suggesting that the substance application has no beneficial effect with respect to serum antioxidant values. In the examinations performed on the tissue TAS averages, it was observed that the alcohol application increased the values significantly compared to the control group. In the alcohol + selenium and the alcohol + ozone + selenium groups, a statistically significant decrease in the tissue value of TAS was found. Besides, the ozone + selenium application in healthy liver tissue had a synergistic effect and increased the tissue TAS values. This indicates that alcohol might increase the antioxidant capacity of the liver, and have a beneficial effect during early stages of consumption. Additionally, only ozone+selenium administration caused a peak in antioxidant levels, leading to the consideration that the protective effect of this combination is dominant over the therapeutic properties.

Concerning the histopathological findings, seleniumsupplemented diet in rats in the form of seleniometionin, prevents the early signs of damage in alcohol-related liver disease developed in the Lieber-DeCarli experimental model (9). Ebselen, an organoselenium compound, has been reported to reduce necrosis, inflammation and fat accumulation in rats treated with alcohol (22). In a study investigating the effects of selenium enriched probiotics on liver fibrosis induced by carbon tetrachloride, selenium was found to suppress hepatic inflammation and reduce hepatic stellate cells by inducing apoptosis (27). In addition, $\mathrm{N}$-acetylcysteine (NAC) and selenium, applied as combination therapy, have been shown to have a marked positive effect on histopathological findings in animals exposed to lead (19). There are no studies investigating the effects of ozone on apoptotic hepatocytes, inflammation, necrosis and steatosis $(32,33)$, which is reported in histopathological analyses in alcoholic liver disease.

In our study, we pointed out that ozone does not have any negative effects on the histopathology of the healthy liver, 
however in the selenium group there was a mild periportal inflammation in 2 rats and a widespread mild hepatocyte injury in 1 rat. These data made us question the usefulness of selenium in healthy liver or that of ozone + selenium treatment. When the alcohol + ozone group was compared to the alcohol group, we observed that the ozone application decreased the severity and prevalence of hepatocyte injury and periportal inflammation, as well as of steatosis. When the alcohol + selenium group was compared to the alcohol group, mild periportal inflammation was observed in many rats. The ozone application seemed to be more effective in terms of periportal inflammation. Concerning hepatocyte injury, it was observed that it decreased the severity and prevalence of the injury and was a better option compared to the alcohol + ozone group. In addition, the alcohol + selenium group reduced the microvesicular steatosis compared to the alcohol group, but failed to reduce it to be comparable to the alcohol + ozone group. When the alcohol + ozone + selenium group was compared to the alcohol group, reduced hepatocyte injury and microvascular steatosis was observed. When the alcohol + ozone + selenium group was compared to the alcohol + ozone group, reduced periportal inflammation was seen, albeit less compared to the alcohol group, whereas compared to the alcohol + selenium group periportal inflammation was reduced more succesfully. The alcohol + ozone + selenium group is superior to both the alcohol + ozone and the alcohol + selenium groups in terms of reducing microvascular steatosis. In a previous study, ozone therapy was shown to prevent liver necrosis in liver damage caused by paracetamol and that, when adjuvant therapy was used as an adjuvant therapy it could lead to improvements in paracetamol toxicity (11). Similarly, Tezcan et al., have reported that ozone can decrease necrosis and inflammation and positively regulate liver regeneration (13).

Collectively, we propose that the most effective agent concerning AST and ALT levels is ozone, while from histopathological evaluations it was ozone or/and selenium that appeared to be a more effective treatment following biopsy analysis. In addition, we suggest that selenium is more effective in cases predominantly with hepatocyte injury and that ozone is more effective in cases predominantly with periportal inflammation, while the combination of ozone + selenium is more effective in cases with steatosis. The results of our findings regarding oxidants and antioxidant evaluations are not in harmony with those of the literature, and this might be related to the fact that the dose of alcohol we applied might have had a positive effect on the oxidant and antioxidant levels in the early stages of liver response, which might, therefore, result in changes in these parameters during later stages.

\section{Conflicts of Interest}

The Authors declare that they have no conflict of interest.

\section{Authors' Contributions}

YA, HAE, MM and GFGconceived and planned the experiments. YA, HAE, MM and GFG carried out the experiments. YA, HAE, MM and GFGcontributed to sample preparation. YA, HAE, MM and GFG contributed to the interpretation of the results. YA took the lead in writing the manuscript. All authors provided critical feedback and helped shape the research, analysis and manuscript.

\section{Acknowledgements}

This study was funded by Kafkas University (2016-TS 39). All applicable international, national, and/or institutional guidelines for the care and use of animals were followed.

\section{References}

1 Torruellas C, French SW and Medici V: Diagnosis of alcoholic liver disease. World J Gastroenterol 20(33): 11684-11699, 2014. PMID: 25206273. DOI: 10.3748/wjg.v20.i33.11684

2 European Association for the Study of the Liver: EASL clinical practical guidelines: management of alcoholic liver disease. J Hepatol 57(2): 399-420, 2012. PMID: 22633836. DOI: 10.1016/ j.jhep.2012.04.004

3 Ceni E, Mello T and Galli A: Pathogenesis of alcoholic liver disease: role of oxidative metabolism. World J Gastroenterol 20(47): 17756-17772, 2014. PMID: 25548474. DOI: 10.3748/ wjg.v20.i47.17756

4 Osna NA, Donohue TM, Jr and Kharbanda KK: Alcoholic liver disease: Pathogenesis and current management. Alcohol Res 38: 147-161, 2017. PMID: 28988570.

5 Crabb DW: Pathogenesis of alcoholic liver disease: newer mechanisms of injury. Keio J Med 48(4): 184-188, 1999. PMID: 10638142.

6 Becker U, Deis A, Sørensen TI, Grønbaek M, Borch-Johnsen K, Müller CF, Schnohr P and Jensen G: Prediction of risk of liver disease by alcohol intake, sex, and age: a prospective population study. Hepatology 23(5): 1025-1029, 1996. PMID: 8621128.

7 Bataller R and Gao B: Liver fibrosis in alcoholic liver disease. Semin Liver Dis 35(2): 146-156, 2015. PMID: 25974900. DOI: 10.1055/s-0035-1550054

8 León Fernández OS, Ajamieh HH, Berlanga $\mathrm{J}$, Menéndez $\mathrm{S}$, Viebahn-Hánsler R, Re L and Carmona AM: Ozone oxidative preconditioning is mediated by $\mathrm{A} 1$ adenosine receptors in a rat model of liver ischemia/reperfusion. Transpl Int 21(1): 39-48, 2008. PMID: 17927680.

9 González-Reimers E, Monedero-Prieto MJ, González-Pérez JM, Durán-Castellón MC, Galindo-Martín L, Abreu-González P, Sánchez-Pérez MJ and Santolaria-Fernández F: Relative and combined effects of selenium, protein deficiency and ethanol on hepatocyte ballooning and liver steatosis. Biol Trace Elem Res 154(2): 281-287, 2013. PMID: 23821313. DOI: 10.1007/s12011013-9734-4

10 Re L, Mawsouf MN, Menéndez S, León OS, Sánchez GM and Hernández F: Ozone therapy: Clinical and basic evidence of its therapeutic potential. Arch Med Res 39(1): 17-26, 2008. PMID: 18067991.

11 Gul H, Uysal B, Cakir E, Yaman H, Macit E, Yildirim AO, Eyi YE, Kaldirim U, Oztas E, Akgul EO, Cayci T, Ozler M, Topal T, 
Oter S, Korkmaz A, Toygar M and Demirbag S: The protective effects of ozone therapy in a rat model of acetaminophen-induced liver injury. Environ Toxicol Pharmacol 34(1): 81-86, 2012. PMID: 22459800. DOI: 10.1016/j.etap.2012.02.006

12 Peralta C, León OS, Xaus C, Prats N, Jalil EC, Planell ES, PuigParellada P, Gelpí E and Roselló-Catafau J: Protective effect of ozone treatment on the injury associated with hepatic ischemiareperfusion: antioxidant-prooxidant balance. Free Radic Res 31(3): 191-196, 1999. PMID: 10499775.

13 Tezcan AH, Ozturk O, Ustebay S, Adali Y and Yagmurdur H: The beneficial effects of ozone therapy in acetaminopheninduced hepatotoxicity in mice. Pharmacol Rep 70(2): 340-345, 2018. PMID: 29477043. DOI: 10.1016/j.pharep.2017.11.003

14 Saeed F, Nadeem M, Ahmed R, Nadeem M, Arshad M and Ullah A: Studying the impact of nutritional immunology underlying the modulation of immune responses by nutritional compounds A review. Food Agric Immunol 27: 205-229, 2016. DOI: 10.1080/09540105.2015.1079600

15 Alpert P: The role of vitamins and minerals on the immune system. Home Health Care Manag Pract 29: 199-202, 2017. DOI: $10.1177 / 1084822317713300$

16 Schulz KF, Altman DG and Moher D: The CONSORT Statement: Revised recommendations for improving the quality of reports of parallel group randomized trials (Turkish translation). Euras J Fam Med 2: 1-10, 2013.

17 Committee for the Update of the Guide for the Care and Use of Laboratory Animals. Guide for the Care and Use of Laboratory Animals, 2011. DOI: 10.17226/12910

18 Singh SP, Padhi PK, Narayan J, Singh A, Pati GK, Nath P, Parida PK and Mishra S: Socioeconomic impact of alcohol in patients with alcoholic liver disease in eastern India. Indian J Gastroenterol 35(6): 419-424, 2016. PMID: 27757779.

19 Sharma S, Raghuvanshi S, Jaswal A, Shrivastava S and Shukla S: Lead acetate-induced hepatoxicity in Wistar rats: possible protective role of combination therapy. J Environ Pathol Toxicol Oncol 34(1): 23-34, 2015. PMID: 25746829.

20 Joshi D, Mittal DK, Shukla S, Srivastav AK and Srivastav SK: $\mathrm{N}$-acetyl cysteine and selenium protects mercuric chlorideinduced oxidative stress and antioxidant defense system in liver and kidney of rats: a histopathological approach. J Trace Elem Med Biol 28(2): 218-226, 2014. PMID: 24485406. DOI: 10.1016/j.jtemb.2013.12.006

21 Messarah M, Klibet F, Boumendjel A, Abdennour C, Bouzerna N, Boulakoud MS and El Feki A: Hepatoprotective role and antioxidant capacity of selenium on arsenic-induced liver injury in rats. Exp Toxicol Pathol 64(3): 167-174, 2012. PMID: 20851583. DOI: 10.1016/j.etp.2010. 08.002

22 Kono H, Arteel GE, Rusyn I, Sies H and Thurman RG: Ebselen prevents early alcohol-induced liver injury in rats. Free Radic Biol Med 30(4): 403-411, 2001. PMID: 11182296.

23 Ajamieh HH, Menéndez S, Merino N, Martínez-sánchez G, Re L and León OS: Ischemic and ozone oxidative preconditioning in the protection against hepatic ischemic-reperfusion injury. Ozone: Sci 25(3): 241-250, 2003. DOI: 10.1080/0191951 0390481568
24 Gultekin FA, Bakkal BH, Guven B, Tasdoven I, Bektas S, Can $\mathrm{M}$ and Comert M: Effects of ozone oxidative preconditioning on radiation-induced organ damage in rats. J Radiat Res 54(1): 3644, 2013. PMID: 22915786. DOI: $10.1093 / \mathrm{jrr} / \mathrm{rrs} 073$

25 Fatima SN and Mahboob T: Role of selenium in protection of liver cirrhosis. Pak J Pharm Sci 26(6): 1097-1102, 2013. PMID: 24191312.

26 Saied NM and Hamza AA: Selenium ameliorates isotretinoininduced liver injury and dyslipidemia via antioxidant effect in rats. Toxicol Mech Methods 24(6): 433-437, 2014. PMID: 24966012. DOI: 10.3109/15376516.2014.937514

27 Liu Y, Liu Q, Ye G, Khan A, Liu J, Gan F, Zhang X, Kumbhar $S$ and Huang K: Protective effects of Selenium-enriched probiotics on carbon tetrachloride-induced liver fibrosis in rats. J Agric Food Chem 63(1): 242-249, 2015. PMID: 25513970.

28 El-Boshy ME, Risha EF, Abdelhamid FM, Mubarak MS and Hadda TB: Protective effects of selenium against cadmium induced hematological disturbances, immunosuppressive, oxidative stress and hepatorenal damage in rats. J Trace Elem Med Biol 29: 104-110, 2015. PMID: 24954678. DOI: 10.1016/ j.jtemb.2014.05.009

29 Ghaffarian-Bahraman A, Shahroozian I, Jafari A and GhaziKhansari M: Protective effect of magnesium and selenium on cadmium toxicity in the isolated perfused rat liver system. Acta Med Iran 52(12): 872-878, 2014. PMID: 25530047.

30 Gultekin FA, Cakmak GK, Turkcu UO, Yurdakan G, Demir FE and Comert M: Effects of ozone oxidative preconditioning on liver regeneration after partial hepatectomy in rats. J Invest Surg 26(5): 242-252. PMID: 23514050. DOI: 10.3109/089419 39.2012.750698

31 Safwat MH, El-Sawalhi MM, Mausouf MN and Shaheen AA: Ozone ameliorates age-related oxidative stress changes in rat liver and kidney: effects of pre- and post-ageing administration. Biochemistry (Mosc) 79(5): 450-458, 2014. PMID: 24954596. DOI: 10.1134/S0006297914050095

$32 \mathrm{Hu}$ S, Yin S, Jiang X, Huang D and Shen G: Melatonin protects against alcoholic liver injury by attenuating oxidative stress, inflammatory response, and apoptosis. Eur J Pharmacol 616(13): 287-292, 2009. PMID: 19576882. DOİ: 10.1016/ j.ejphar. 2009.06.044.

33 Mishra A, Paul S and Swarnakar S: Downregulation of matrix metalloproteinase- 9 by melatonin during prevention of alcoholinduced liver injury in mice. Biochimie 93(5): 854-866, 2011. PMID: 21354255. DOI: 10.1016/j.biochi.2011.02.007
Received February 24, 2019

Revised April 3, 2019

Accepted April 9, 2019 